\title{
RIGHTIDEALS IN $\Gamma$-SEMIGROUPS
}

\author{
V. Jyothi ${ }^{\S}$, Y. Sarala ${ }^{2}$, Rao T. Nageshwara ${ }^{3}$, Rao D. Madhusudhana ${ }^{4}$ \\ ${ }^{1}$ Department of Mathematics \\ K.L. University \\ Guntur Dt., A.P., INDIA \\ ${ }^{2}$ Department of Mathematics \\ NIT A.P., K.L. University \\ Guntur Dt., A.P., INDIA
}

\begin{abstract}
In This article to prove an equivalent of the krull- intersection theorem $\cap_{m=1}^{\infty} M^{m}=$ 0 in 2.11 and thus it is shown that the $\Gamma$ - semigroup with the krull - intersection property is nearly a principal $\Gamma$ - rightideal $\Gamma$ - semigroup. Every principal $\Gamma$ - rightideal $\Gamma$ - semigroup need not have the krull - intersection property and so it is researched what type of principal $\Gamma$ - rightideal $\Gamma$ - semigroups, not vitally containing an identity, have this intersection property. It is initiate in 2.11, 2.13 and 3.1, that the equivalent of the krull - intersection theorem is true under some additional conditions unlike in ring theory.
\end{abstract}

AMS Subject Classification: 20M10, 20M99

Key Words: principal $\Gamma$ - rightideal, $\Gamma$ - right Noetherian, $\Gamma$ - right uniform

\section{Introduction}

A $\Gamma$-semigroup $S$ is called a principal $\Gamma$-rightideal $R$ is principal. Naturally one may ask whether it is possible to determine the structure of $S$ by specializing some property of $M$. By virtue of the existence of the unique maximal $\Gamma$-rightideal. The influenced the author to prove an analogue of the krull inter-

Received: $\quad$ December 17, 2016

Revised: May 20, 2017

Published: $\quad$ May 23, 2017

(c) 2017 Academic Publications, Ltd. url: www.acadpubl.eu

$\S_{\text {Correspondence author }}$ 
section theorem $\left(\cap_{m=1}^{\infty} M^{m}=0\right)$ and thus it is shown that the $\Gamma$-semigroup with the krull intersection property is nearly a principal $\Gamma$-rightideal $\Gamma$-semigroup.

\section{Main Results}

Definition 2.1. A $\Gamma$ - semigroup $S$ is called a principal $\Gamma$ - rightideal if every right $\Gamma$ - ideal $R$ is principal. i.e $R=f \cup f \Gamma s$ for some $f \in S$. Since the $\Gamma$-rightideals in a principal $\Gamma$ - rightideal of $S$ form a chain under set inclusion, $S$ is either right simple or has a unique maximal proper $\Gamma$ - right ideal $M$.

Theorem 2.2. If $S$ is a principal $\Gamma$ - rightideal in $\Gamma$-semigroup, then the $\Gamma$ - rightideals form a chain under set inclusion.

Proof. If $P$ and $Q$ are any two $\Gamma$ - rightideals then $P \cup Q=e \cup e \Gamma S$ for some $e \in S$.

Since $e \in P$ or $e \in Q$, we must have $P \subseteq Q$ or $Q \subseteq P$.

Remark 2.3. The above theorem is not true. Suppose that $S$ is not principal.

Example 2.4. Let us assume that $S=\{0, p, q\}$ subject to the condition $p^{2}=p ; q^{2}=p \alpha q=q \alpha p=0 ; \alpha \in \Gamma$ and $0 \beta s=s \beta 0=0$ for each $s \in S, \beta \in \Gamma$.

Clearly $(0, a) \cap(0, b)=0$ and excepts $S$ every other $\Gamma$-ideal is principal.

We shall now indicate the nature of elements of $S$, if $S$ is a right Noetherian $\Gamma$ - semigroup fulfills the property of being right uniform, and derive from this the nature of elements of principal rightideals in $\Gamma$-semigroups.

Definition 2.5. A $\Gamma$-semigroup $S$ is called a $\Gamma$-right Noetherian if $S$ fulfills ascending chain conditions on $\Gamma$-rightideals.

Definition 2.6. A $\Gamma$ - semigroup $S$ is called a $\Gamma$-rightuniform if every $\Gamma$ rightideal has nonempty intersection with every other $\Gamma$ - right ideal.

If $S$ has zero, this intersection should be nonzero. For any subsets $B$ of a $\Gamma$ - semigroup $S$ we denote $B^{R}=\{x \in S / B \Gamma x=0\}$.

Lemma 2.7. Let $S$ be a $\Gamma$ - right Noetherian semigroup including zero. If $b^{R} \neq 0$, then either $b$ is nilpotent or $\exists$ a positive integer $k \ni\left(b^{k}\right)^{R} \cap\left(b^{k} \Gamma S \cup b^{k}\right)=$ 0 .

Proof. By $\Gamma$ - right Noetherian condition, the ascending chain of $\Gamma$ rightideals $b^{R} \subset\left(b^{2}\right)^{R} \subset \cdots$ teriminates. $\exists$ a positive integer $k \ni\left(b^{k}\right)^{R}=$ $\left(b^{k+1}\right)^{R}=\cdots$.

Let $x \in\left(b^{k}\right)^{R} \cap\left(b^{k} \Gamma S \cup b^{k}\right)$. 
If $x=b^{k}, b^{2 k}=0$ and hence $b$ is nilpotent.

If $x \neq b^{k} ; x=b^{k} \alpha y$ and also $b^{k} \alpha x=0 ; \alpha \in \Gamma$.

This implies that $b^{2 k} \alpha y=0$ and so $y \in\left(b^{2 k}\right)^{R}=\left(b^{k}\right)^{R}$. Thus $b^{k} \alpha y=x=0$; $\alpha \in \Gamma$. As an easy consequence of 2.2 and 2.6, we receive the next result.

Theorem 2.8. Let $S$ be a $\Gamma$-semigroup including zero. Then, for any $b \in S$ , either $b^{R}=0$ or $b$ is nilpotent if $S$ fulfills either of the following conditions:

a) $S$ is a $\Gamma$-right Noetherian and $\Gamma$-right uniform.

b) $S$ is a principal $\Gamma$-right ideal $\Gamma$-semigroup.

Remark 2.9. The theorem 2.8 fails if we leave the uniform condition from (a). Also 2.8 is not true if we suppose that every $\Gamma$-rightideal exclude $S$ is principal in the condition (b). Both these facts can be shown by the example 2.4 .

Let $S$ be a principally generated $\Gamma$-semigroup; i.e $S$ is of the form $e \cup e \cup \Gamma S$ where $e \in S$. Since $S$ is not right simple, therefore $S$ has a unique maximal proper $\Gamma$-rightideal $M$, which is the union of all $\Gamma$-rightideals of $S \nsubseteq e$. If $S \Gamma M \neq S$, then $S \Gamma M \subseteq M$ and hence $M$ is a $\Gamma$-ideal. In this case $M=b \cup b \Gamma S$, then it is simple to show that $M^{n}=b^{n} \cup b^{n} \Gamma S$ for each positive integer $n$. However $\cap_{n=1}^{\infty} M^{n}$ need not be empty. If $S \Gamma M=S$ and $M=b \cup b \Gamma S$, then it can be simply verified that $M^{n}=b \Gamma S$ for each positive integer $n>1$, which implies that $\cap_{n=1}^{\infty} M^{n} \neq \emptyset$. But if $\cap_{n=1}^{\infty} M^{n}=0$ need not be empty. If $S \Gamma M=S$ and $M=b \cup b \Gamma S$, then it can be simply proved that $M^{n}=b \Gamma S$ for every positive integer $n>1$ which implies that $\cap_{n=1}^{\infty} M^{n} \neq \phi$. But if $\cap_{n=1}^{\infty} M^{n}=\phi$ it will be observed below that $S$ is nearly principal $\Gamma$ - right ideal $\Gamma$ - semigroup.

Notation 2.10. Let $(S, \Gamma, M)$ assigned a $\Gamma$ - semigroup with a unique maximal right ideal $M$. Throughout this paper we suppose that $M$ exists.

Theorem 2.11. Let $(S, \Gamma, M)$ be a $\Gamma$ - semigroup such that $S=e \cup e \Gamma S$ and $M=b \cup b \Gamma S$. Then $\cap_{m=1}^{\infty} M^{m}=\phi$ if and only if:

i) every $\Gamma$-rightideal is either $b^{m} \Gamma S$ or $\left(b^{m} \Gamma S \cup b^{n}\right)$;

ii) $M^{p} \neq M^{q}$ for any pair of natural numbers $p$ and $q$ and $p \neq q$;

iii) $S \Gamma M \neq S$.

If $S$ has zero and $M^{r}$ is zero then $\cap_{m=1}^{\infty} M^{m}=0$ and this $\Gamma$-idealstructure of $S$ is as represented above.

Proof. Let $B$ be a proper $\Gamma$ - rightideal. Then $B \subseteq M$. 
Assume that $\cap_{m=1}^{\infty} M^{m}=\emptyset$. Then there exist a least natural number $r$ such that $B \subseteq M^{r}$ and $B \nsubseteq M^{r+1}$, i.e. $\exists$ an $y \in B \ni y=b^{r}$ or $y=b^{r} \alpha x$ where $x \notin M ; \alpha \in \Gamma$. If $y=b^{r}$, then $B=b^{r} \cup b^{r} \alpha S$.

In the second case $x \notin M$ implies $x \cup x \Gamma S=S$. Now $y=b^{r} \alpha x$ and so $y \Gamma S=b^{r} \alpha x \Gamma S=b^{r} \Gamma(S \backslash x)=b^{r} \Gamma S \backslash b^{r} \alpha x$. Since $y \Gamma S \subseteq B, b^{r} \Gamma S \backslash b^{r} \alpha x \subseteq B$. But $b^{r} \alpha x \in B$. Hence $b^{r} \Gamma S \subseteq B \subseteq b^{r} \Gamma S \cup b^{r}$. Thus $B=b^{r} \Gamma S$ or $b^{r} \Gamma S \cup b^{r}$.

If $\cap_{m=1}^{\infty} M^{m}=\phi$, then the condition (ii) is obviously satisfied. Further more, from the introduction of this section, the condition (iii) is clear. Conversely, let $\cap_{m=1}^{\infty} M^{m}$ be non-empty.

If $\cap_{m=1}^{\infty} M^{m}=b^{m} \Gamma S \cup b^{m}$, then $M^{m}=M^{m+1}$, which is a contradiction.

If $\cap_{m=1}^{\infty} M^{m}=b^{m} \Gamma S$, then $M^{m+1}=b^{m} \Gamma S$. Hence $M^{m+1}=M^{m+2}$ which is a contradiction.

The remainder of the proof follows from the first part.

Corollary 2.12. If $(S, \Gamma, M)$ is a $\Gamma$-semigroup with identity and $M=b \Gamma S$, then $\cap_{m=1}^{\infty} M^{m}$ is empty if and only if:

(i) every $\Gamma$-rightideal is of the form $M^{r}$.

(ii) $M^{p} \neq M^{q}$ for natural number $p$ and $q$ and $p \neq q$.

(iii) $S \Gamma M \neq S$.

Not every principal $\Gamma$ - right ideal $\Gamma$ - semigroup $(S, \Gamma, M)$ satisfies the property that $\cap_{m=1}^{\infty} M^{m}$ is empty. For, let $S=\left\{b, b^{2}, b^{3}=b^{4}\right\}$ or $S=\left\{1, b, b^{2}=b^{3}\right\}$.

The following theorem gives a class of principal $\Gamma$-rightideal $\Gamma$-semigroups with the above conditions.

Theorem 2.13. Let $(S, \Gamma, M)$ be a left Noetherian $\Gamma$ - semigroup without identity such that $S \Gamma M \neq S, S=e \cup e \Gamma S$ and $M=b \cup b \Gamma S$. Then $\cap_{m=1}^{\infty} M^{m}=\phi$ (and hence the only $\Gamma$-right ideals of $\mathrm{S}$ are either $M^{r}$ or $b^{r} \Gamma S$ ) if either of the following condition is fullfilled:

(i) there exists no $p$ and $q$ in $S$ such that $p=q \alpha p$ or $p=p \alpha q$.

(ii) $S$ is $\Gamma$-cancellative and $S$ has no $\Gamma$-idempotents.

Proof. Since $S \Gamma M \neq S, M$ is an ideal and so $M^{m}=b^{m} \cup b^{m} \Gamma S$. Assume that $\cap_{m=1}^{\infty} M^{m} \neq \phi$. Then $p=b \alpha t_{1}=b^{2} \alpha t_{2}=\cdots$. By virtue of the condition (i) and (ii) it is clear that $t_{i} \neq t_{j}$. Then by $\Gamma$-left Noetherian condition the chain $s_{1} \cup S \Gamma t_{1} \subset s_{2} \cup S \Gamma t_{2} \subset \cdots$ teriminates. So $p=b^{n} \alpha t_{n}$, where $t_{n} \notin M$. Then $t_{n}=t_{n} \cup t_{n} \Gamma S=S$ which implies that $p \Gamma S=b^{n} \alpha t_{n} \Gamma S=b^{n} \alpha\left(S \backslash t_{n}\right)=$ $b^{r} \alpha S \backslash b^{n} \alpha t_{n}$. Hence $p \cup p \Gamma S=b^{n} \Gamma S$. Since $b^{2 n} \in M^{2 n+1}$. Hence $b^{2 n}=b^{2 n+1}$ or $b^{2 n}=b^{2 n+1} \alpha s$, which implies that the condition (i) can not fulfilled. 
Assume condition (ii), evidently $b^{2 n} \neq b^{2 n+1}$. Then $b^{2 n}=b^{2 n+1} \alpha s$ implies that $b=b^{2} \alpha s$ by the cancellative condition. Now $a \in M$ implies $a=b^{t} \alpha q$ where $q \notin M$, by the $\Gamma$ - left Noetherian condition ( otherwise $a=b \alpha q, q \in M$ then $q=a b \alpha q_{1} \cdots$ and so we have an ascending chain of $\Gamma$-left ideals $q \cup S \Gamma q \subset$ $\left.q_{1} \subset S \Gamma q_{1} \subset \cdots\right)$ clearly $b^{t} \alpha q=b=b^{t-1} \alpha\left(b^{2} \beta s\right) r q=b^{t} \alpha(b \beta s r q)$. Since $q \notin M$, $q \cup q \cup \Gamma S=S$. Then $b \alpha s \beta q=q$ or $b \alpha s \beta q=q \alpha m$ where $m \in S ; \alpha, \beta \in \Gamma$. The first equality is not possible, since otherwise $q \in M$, a contradiction. Thus we have $b^{t} \alpha q=b^{t} \alpha q \beta m$. Then by the cancellative condition, $q=q \alpha m$ and so $q \alpha m=q \alpha m^{2}$. Again by the cancellative condition, $m=m^{2}$, which is a contradiction by hypothesis.

Remark 2.14 The theorem 2.13 fails if we drop the condition that $S$ is cancellative in (i). Note the example where $S=\left\{b, b^{2}, b^{3}=b^{4}\right\}$.

Note 2.15. In 2.13 condition (ii) always implies condition (i). The author does't know whether these two condition are equivalent.

\section{Principal $\Gamma$-Rightideal $\Gamma$-Semigroups with Identity}

In 2.12 we considered conditions under which $\cap_{m=1}^{\infty} M^{m}=\phi$, for a principal $\Gamma$ right ideal $\Gamma$-semigroup $(S, \Gamma, M)$ with identity. In 2.13 we obtained a class of principal right ideal $\Gamma$-semigroups without identity having the krull- intersection property.

The purpose of this section is to show that theorem 2.13 can be proved with a weaker hypothesis if the $\Gamma$-semigroup has identity.

Theorem 3.1. Let $(S, \Gamma, M)$ be a left Noetherian $\Gamma$-semigroup with identity suppose $M=b \Gamma S$ and $S$ satisfies either of the following conditions:

(i) $S$ is concellative.

(ii) there exists no element $q$ in $S$ such that $b \alpha q=q$ and $M^{x} \neq M^{y}$ for all positive integer $x$ and $y$ and $x \neq y$.

Then every element $m \in M$ is of the form $b^{x} \alpha q, x$ being a positive integer and $q$ is a left union in $S$. Every $\Gamma$-rightideal is of the form $b^{t} \Gamma S$. If $S \Gamma M \neq S$, then every $m \in M$ is of the form $b^{x} \alpha q$ where $q$ is a unit of $S$. Also every $\Gamma$-rightideal of $S$ is of the form $M^{t}$ and $\cap_{m=1}^{\infty} M^{m}=\emptyset$.

Proof. Let $m \in M$. Then $m=b \alpha t_{1}$. Evidently $t_{1} \neq m$ by condition (i). Condition (ii) also implies $t_{1} \neq m$, since otherwise $m=b \alpha m$ implies $1=b$ by the cancellative property. If $t_{1} \in M$, then $t_{1}=b \alpha t_{2}$. Proceeding in this manner, we obtain an ascending chain of $\Gamma$ - leftideals $S \Gamma t_{1} \subset S \Gamma t_{2} \subset \cdots$ by 
the left Noetherian condition this chain teriminates. Hence $m=b^{x} \alpha q, q \notin M$, $\alpha \in \Gamma$. Since every $\Gamma$-rightideal is included in the unique maximal $\Gamma$-rightideal $M$, we have $q \alpha t=1$, i.e $q$ is a $\Gamma$-left unit. Let $A$ be a $\Gamma$-rightideal. If $r \in A$, $r=b^{x} \alpha q$ where $q \alpha t=1$. Hence $b^{x}=r \alpha t \in A$. Let $k=\min \left\{x / b^{x} \in A\right\}$. Then $A=b^{k} \Gamma S$.

If $S \Gamma M \neq S$, then $M$ is an ideal and so $M^{t}=b^{t} \Gamma S$ for every $t$. Furthermore every $r \in M$ is of the form $b^{x} \alpha q$. $q$ must be a unit. For since $q \notin M$ and so $S \Gamma q \not \subset M$. Hence $S \Gamma q=S$, which implies $t \alpha q=1$.

If $\cap_{m=1}^{\infty} M^{m} \neq \emptyset$, then $\cap_{m=1}^{\infty} M^{m}=M^{t}$ and so $M^{t}=M^{t+1}=\cdots$ This leads to a contradiction by condition (ii). Also by condition (i) $M^{t}=M^{t+1} \Rightarrow b^{t}=$ $b^{t+1} \alpha y \Rightarrow 1=b \alpha y \Rightarrow M=S$ a contradiction.

Remark 3.2. The hypothesis that there exist no $r$ such that $b \alpha r=r$ is essential in 3.1 .

Example 3.3. For, condition the $\Gamma$ - semigroup $\{a, x, y, 1\}$ with the multiplication table

\begin{tabular}{l|lllll}
$\bullet$ & $a$ & $x$ & $y$ & 1 \\
\hline$a$ & $a$ & $a$ & $a$ & $a$ \\
$x$ & $a$ & $x$ & $x$ & $x$ \\
$y$ & $a$ & $x$ & $x$ & $y$ \\
1 & $a$ & $x$ & $y$ & 1
\end{tabular}

In this $\Gamma$ - semigroup the rightideals are $y \Gamma S, y^{2} \Gamma S$ and $a \Gamma S$ and the unique maximal rightideal is $y \Gamma S$; $y \alpha a=a$ and $a \neq y^{n}$ for any $n$.

Remark 3.3. Let us give an example $[1 ; 109]$ illustrating the theorem for the $\Gamma$ - semigroup $(S, \Gamma, M)$ such that $S \Gamma M=S$. Let $S=\{1, x, y\}$ subject to the condition $x \alpha y=1$. Then $M=y \Gamma S$ and there exist no $b$ in $S$ such that $y \alpha b=b$. Also $S$ is cancellative. Every element of $S$ is of the form $y^{i} x^{i}$ where $i, j=0,1,---; x^{0}=y^{0}=1$ and every $\Gamma$-rightideal is of the form $y^{i} \Gamma S$. However $M^{t}=M=x \Gamma S$ forevery positive integer $t$.

Theorem 3.4. Let $M$ be a nilpotent and $M=a \Gamma S$ in a $\Gamma$ - semigroup $(S, \Gamma, M)$ with zero and identity. Then $\cap_{n=1}^{\infty} M^{n}=0$. If $S \Gamma M=S$, then $S$ is right simple, while if $S \Gamma M \neq S$, then $S=G \cup M$, where $G$ is the group of units of $S$ and $M=\left\{a^{r} \alpha g: 1 \leq r \leq t ; g \in G\right.$ and $t$ is the index of nilpotency of $\left.M\right\}$

Proof. Clearly $\cap_{m=1}^{\infty} M^{m}=0$. If $S \Gamma M=S$, then $0=M^{t}=M$ and hence $S$ is right simple.

To prove the last part: $x \neq 0 ; x \neq a^{r}$ and $x \in M \Rightarrow x=a \alpha t . t \neq x$ since otherwise, $x=a \alpha x \Rightarrow a^{t-1} \alpha x=0 \Rightarrow a^{t-2} \alpha x=0 \Rightarrow---\Rightarrow a \alpha x=0 \Rightarrow$ 
$x=0$. If $t$ is not a unit, $t=a \alpha S$ and thus $x=a^{2} \alpha S$. By proceeding in this manner, since $a^{t}=0$, we must have $x=a^{r} \alpha y$, where $y$ is a unit.

\section{References}

[1] A.H. Clliford and G.B. Preston, The Algebraic theory of semigroups, Math. Surveys, Amer. Math. Society, 1, No. 7 (1967).

[2] M. Satyanarayana, Principal right ideal semigroups, J. London Math. Soc., 3, No. 2 (1971), 549-553. 
